HGU-CAP-021

\title{
Isospin violating dark matter being asymmetric
}

\author{
Nobuchika Okada* \\ Department of Physics and Astronomy, \\ University of Alabama, Tuscaloosa, Alabama 35487, USA \\ Osamu Setdt \\ Department of Life Science and Technology, \\ Hokkai-Gakuen University, Sapporo 062-8605, Japan
}

\begin{abstract}
The isospin violating dark matter (IVDM) scenario offers an interesting possibility to reconcile conflicting results among direct dark matter search experiments for a mass range around $10 \mathrm{GeV}$. We consider two simple renormalizable IVDM models with a complex scalar dark matter and a Dirac fermion dark matter, respectively, whose stability is ensured by the conservation of "dark matter number." Although both models successfully work as the IVDM scenario with destructive interference between effective couplings to proton and neutron, the dark matter annihilation cross section is found to exceed the cosmological/astrophysical upper bounds. Then, we propose a simple scenario to reconcile the IVDM scenario with the cosmological/astrophysical bounds, namely, the IVDM being asymmetric. Assuming a suitable amount of dark matter asymmetry has been generated in the early Universe, the annihilation cross section beyond the cosmological/astrophysical upper bound nicely works to dramatically reduce the antidark matter relic density and as a result, the constraints from dark matter indirect searches are avoided. We also discuss collider experimental constraints on the models and an implication to Higgs boson physics.
\end{abstract}

*Electronic address: okadan@ua.edu

${ }^{\dagger}$ Electronic address: seto@physics.umn.edu 


\section{INTRODUCTION}

Light weakly interacting massive particles (WIMPs) with a mass around $10 \mathrm{GeV}$ have been currently a subject of interest, motivated by some recent results in direct dark matter (DM) detection experiments. DAMA/LIBRA has claimed detections of the annual modulation signal by WIMPs [1]. CoGeNT has found an irreducible excess [2] and annual modulation [3]. CRESST has observed many events that expected backgrounds are not enough to account for [4, 5]. However, these observations are challenged to the null results obtained by other experimental collaborations, CDMS [6], XENON10 [7], XENON100 [8, 9], and SIMPLE [10].

Light WIMPs have been investigated for a dark matter interpretation of those data. For instance, very light neutralino in the minimal supersymmetric standard model (MSSM) 11, 12] and the next-to-MSSM (NMSSM) [13, 14] or very light right-handed sneutrino [15, 16] in the NMSSM. On the other hand, the Fermi-LAT Collaboration has derived constraints on an $s$-wave annihilation cross section of a WIMP based on the analysis of gamma ray flux [17]. Annihilation modes of a light WIMP is now severely constrained.

The isospin violating dark matter (IVDM) [18] has been proposed as a way to reconcile the tension between inconsistent results among the direct DM detection experiment, since different nuclei for target material have been used in the detector of each experiments. The possible consistency between DAMA, CoGeNT [2], and XENON [7, 8] was pointed out [18], while the discrepancy between CoGeNT and CDMS cannot be resolved by IVDM because both of them use germanium as the target. However, recently it was reported [19] that CDMS-II Si have observed three events and its possible signal region overlaps with the possible CoGeNT signal region analyzed by Kelso et al. [20]. The fitting data with IVDM have been examined by several groups [20 28], and constraints from indirect [29] and direct [30] DM detection experiments also have been derived.

In this paper, we consider two simple IVDM models with a complex scalar DM and a Dirac fermion DM, respectively. In most of the previous works, the IVDM models have been proposed by introducing a new $U(1)$ gauge symmetry with $Z^{\prime}$ boson [31 33] or an extension of the Higgs sector [32, 34, 35]. In contrast to those models, to realize the different cross sections with respect to up quarks and down quarks, we introduce fourth generation quarks in the scalar DM model and scalar quarks in the fermion DM model, respectively. Our 
TABLE I: Particle contents for the model S

\begin{tabular}{|c|c|c|c|c|}
\hline Fields & $S U(3)_{c}$ & $S U(2)_{L}$ & $U(1)_{Y}$ & $U(1)_{G}$ \\
\hline \hline$U$ & $\mathbf{3}$ & $\mathbf{1}$ & $+2 / 3$ & +1 \\
\hline$D$ & $\mathbf{3}$ & $\mathbf{1}$ & $-1 / 3$ & +1 \\
\hline$\phi$ & $\mathbf{1}$ & $\mathbf{1}$ & 0 & +1 \\
\hline$S$ & $\mathbf{1}$ & $\mathbf{1}$ & 0 & 0 \\
\hline
\end{tabular}

models are similar to a model briefly mentioned in Ref. [18].

The paper is organized as follows. In the next section, we describe our models of scalar and fermion DMs. In Sec. III, we identify the allowed region of the mass and couplings of the mediator quarks or scalar quarks by imposing the condition of the isospin violating elastic scattering cross section with nuclei. In Sec. IV, we calculate the annihilation cross section of the IVDM to examine the resultant thermal relic density as well as the constraint from Fermi-LAT data for the parameter region found in Sec. III. Constraints from collider experiments are discussed in Sec. V. Section VI is devoted to conclusions.

\section{MODELS}

\section{A. Model of scalar dark matter with fermion mediators (model S)}

First, we consider a simple model with a complex scalar dark matter, whose particle contents are given in Table I. In addition to the Standard Model (SM) particle contents, we have introduced the SM $S U(2)$ singlet Dirac fermions ( $U$ and $D)$ whose representations are the same as $S U(2)$ singlet up and down quarks, a complex scalar DM $(\phi)$, and a real scalar $S$, with a global $U(1)_{G}$ symmetry. The stability of $\phi$ is ensured by the global $U(1)_{G}$ symmetry assumed to be conserved. All the SM particles are neutral under the global symmetry.

The gauge and global symmetric Lagrangian relevant to our discussion is given by

$$
\mathcal{L} \supset-M_{U} \bar{U} U-M_{D} \bar{D} D-\left(f_{U} \overline{U_{L}} \phi u_{R}+f_{D} \overline{D_{L}} \phi d_{R}+\text { H.c. }\right)-V(H, \phi, S),
$$

where $H$ is the SM Higgs doublet, $u_{R}\left(d_{R}\right)$ is the SM right-handed up (down) quark singlet, and $V$ is a scalar potential for $H, \phi$, and $S$. 
We assume a suitable scalar potential for our discussion: not only the Higgs doublet but also the scalar $S$ develop vacuum expectation values and we expand these scalar fields as

$$
H=\left(\begin{array}{c}
0 \\
\frac{1}{\sqrt{2}}(v+h)
\end{array}\right), \quad S=v_{s}+s,
$$

with the vacuum expectation values, $v=246 \mathrm{GeV}$ and $v_{s}$.

After the electroweak symmetry breaking, the SM singlet scalar and the Higgs boson have a mass mixing such that

$$
\left(\begin{array}{l}
s \\
h
\end{array}\right)=\left(\begin{array}{cc}
\cos \alpha & \sin \alpha \\
-\sin \alpha & \cos \alpha
\end{array}\right)\left(\begin{array}{l}
h_{1} \\
h_{2}
\end{array}\right),
$$

where $h_{1}$ and $h_{2}$ are the mass eigenstates with masses $m_{h_{1}} \leq m_{h_{2}}$, respectively. The existence of a light scalar particle mixed with the SM Higgs boson is constrained by the LEP experiments [36, 37]. We consider a small mixing, for example, $\sin \alpha<0.1$, so that the mass eigenstate $h_{1}\left(h_{2}\right)$ is almost the SM singlet scalar (the SM Higgs boson). For such a small mixing, the lower mass bound on $h_{1}$ disappears, and in the following analysis we consider $m_{h_{1}}<10 \mathrm{GeV}$. Terms in the scalar potential relevant to our analysis below are triple scalar couplings parametrized as

$$
V \supset v\left(\lambda_{1} h_{1}+\lambda_{2} h_{2}\right) \phi^{\dagger} \phi+\lambda_{3} v h_{1}^{2} h_{2},
$$

with dimensionless couplings $\lambda_{1,2,3}$. Since the SM-like Higgs boson $h_{2}$ can decay to the lighter scalars, $h_{1}$ and $\phi$ ( $h_{1}$ subsequently decays to lighter SM particles), the couplings $\lambda_{2,3}$ should be small in order not to significantly alter the Higgs boson branching ratio from the SM prediction. To simplify our analysis, we assume $\lambda_{2} \gg \lambda_{3}$ and further parametrize $\lambda_{1,2}$

as $\lambda_{1}=\lambda \cos \alpha$ and $\lambda_{2}=\lambda \sin \alpha$ with $\lambda=\sqrt{\lambda_{1}^{2}+\lambda_{2}^{2}}$. We will discuss a phenomenological constraint on these parameters from the invisible decay branching ratio of the SM Higgs boson in Sec. $\mathrm{VB}$,

\section{B. Model of fermion dark matter with scalar mediators (model F)}

Next, we consider a simple model with a Dirac fermion DM, whose particle contents are given in Table III. In addition to the SM particle contents, we introduce color triplet scalars $\left(\tilde{Q}_{L}, \tilde{U}_{R}\right.$, and $\left.\tilde{D}_{R}\right)$ that are analogous to the scalar quarks in the MSSM, and a Dirac 
TABLE II: Particle contents for the model F

\begin{tabular}{|c|c|c|c|c|}
\hline Fields & $S U(3)_{c}$ & $S U(2)_{L}$ & $U(1)_{Y}$ & $U(1)_{G}$ \\
\hline \hline$\tilde{Q}_{L}$ & $\mathbf{3}$ & $\mathbf{2}$ & $+1 / 6$ & +1 \\
\hline$\tilde{U}_{R}$ & $\mathbf{3}$ & $\mathbf{1}$ & $+2 / 3$ & +1 \\
\hline$\tilde{D}_{R}$ & $\mathbf{3}$ & $\mathbf{1}$ & $-1 / 3$ & +1 \\
\hline$\psi$ & $\mathbf{1}$ & $\mathbf{1}$ & 0 & -1 \\
\hline
\end{tabular}

fermion DM $(\psi)$. Similarly to the model S, a global $U(1)_{\mathrm{G}}$ symmetry has been introduced to ensure the stability of the Dirac fermion DM. All the SM fields are neutral under the global symmetry.

The relevant part of the Lagrangian is given by

$$
\begin{aligned}
\mathcal{L} \supset & -m_{\psi} \bar{\psi} \psi-M_{Q}^{2} \tilde{Q}_{L}^{\dagger} \tilde{Q}_{L}-M_{U}^{2} \tilde{U}_{R}^{\dagger} \tilde{U}_{R}-M_{D}^{2} \tilde{D}_{R}^{\dagger} \tilde{D}_{R} \\
& +A_{U} \tilde{Q}_{L}^{\dagger} \tilde{H} \tilde{U}_{R}+A_{D} \tilde{Q}_{L}^{\dagger} H \tilde{D}_{R}+\text { H.c. } \\
& -f_{L} \bar{\psi} \tilde{Q}_{L}^{\dagger} q_{L}-f_{R_{u}} \bar{\psi} \tilde{U}_{R}^{\dagger} u_{R}-f_{R_{d}} \bar{\psi} \tilde{D}_{R}^{\dagger} d_{R}+\text { H.c. }
\end{aligned}
$$

where $\tilde{H}=i \sigma_{2} H^{*}, \tilde{Q}_{L}=\left(\tilde{U}_{L} \tilde{D}_{L}\right)^{T}, q_{L}=\left(u_{L} d_{L}\right)^{T}$ is the SM doublet quark of the first generation, and $A_{U, D}$ are parameters with mass-dimension one.

After the electroweak symmetry breaking, the mass eigenstates of $\tilde{U}$ are obtained as

$$
\left(\begin{array}{c}
\tilde{U}_{L} \\
\tilde{U}_{R}
\end{array}\right)=\left(\begin{array}{cc}
\cos \theta_{u} & \sin \theta_{u} \\
-\sin \theta_{u} & \cos \theta_{u}
\end{array}\right)\left(\begin{array}{c}
\tilde{U}_{1} \\
\tilde{U}_{2}
\end{array}\right),
$$

with a mixing angle $\theta_{u}$. Similarly, $\tilde{D}_{1}$ and $\tilde{D}_{2}$ are obtained with an angle $\theta_{d}$. With the mass eigenstates, the Yukawa interactions between the dark matter fermion and the SM quarks in Eq. (5) are rewritten as

$$
\begin{aligned}
\mathcal{L}_{\mathrm{Y}} & =-\bar{\psi}\left(f_{L} \cos \theta_{u} P_{L}-f_{R_{u}} \sin \theta_{u} P_{R}\right) \tilde{U}_{1}^{\dagger} u-\bar{\psi}\left(f_{L} \sin \theta_{u} P_{L}+f_{R_{u}} \cos \theta_{u} P_{R}\right) \tilde{U}_{2}^{\dagger} u \\
& -\bar{\psi}\left(f_{L} \cos \theta_{d} P_{L}-f_{R_{d}} \sin \theta_{d} P_{R}\right) \tilde{D}_{1}^{\dagger} d-\bar{\psi}\left(f_{L} \sin \theta_{d} P_{L}+f_{R_{d}} \cos \theta_{d} P_{R}\right) \tilde{D}_{2}^{\dagger} d+\text { H.c. }
\end{aligned}
$$




\section{DARK MATTER ELASTIC SCATTERING WITH NUCLEI}

The dark matter scattering cross section with nucleus $(N)$ made of $Z$ protons $(p)$ and $A-Z$ neutrons $(n)$ is given by

$$
\sigma_{\mathrm{SI}}^{N}=\frac{1}{\pi}\left(\frac{m_{N}}{m_{N}+m_{\phi}}\right)^{2}\left(Z f_{p}+(A-Z) f_{n}\right)^{2},
$$

for a scalar dark matter, while for a Dirac fermion dark matter

$$
\sigma_{\mathrm{SI}}^{N}=\frac{1}{\pi}\left(\frac{m_{N} m_{\psi}}{m_{N}+m_{\psi}}\right)^{2}\left(Z f_{p}+(A-Z) f_{n}\right)^{2} .
$$

The effective coupling with a proton $f_{p}$ and a neutron $f_{n}$ is expressed, by use of the hadronic matrix element, as

$$
\frac{f_{i}}{m_{i}}=\sum_{q=u, d, s} f_{T q}^{(i)} \frac{\alpha_{q}}{m_{q}}+\frac{2}{27} f_{T G}^{(i)} \sum_{c, b, t} \frac{\alpha_{q}}{m_{q}},
$$

where $\alpha_{q}$ is an effective coupling of the DM particle with a $q$-flavor quark defined in the operators

$$
\mathcal{L}_{\text {int }}=\left\{\begin{array}{ll}
\alpha_{q} \bar{q} q|\phi|^{2} & \text { for } \quad \phi \\
\alpha_{q} \bar{q} q \bar{\psi} \psi & \text { for } \psi
\end{array},\right.
$$

with its mass $m_{q}, f_{T q}^{(i)}$, and $f_{T G}^{(i)}$ where $i=p, n$ are constants. In our analysis, we use the following values: $f_{T u}^{(p)}=0.0290, f_{T d}^{(p)}=0.0352, f_{T u}^{(n)}=0.0195, f_{T d}^{(n)}=0.0525, f_{T s}^{(i)}=0$, and $f_{T G}^{(i)}=1-\sum_{q=u, d, s} f_{T q}^{(i)}$. Those $f_{T u}^{(i)}$ and $f_{T d}^{(i)}$ are quoted from Ref. [38], while we set $f_{T s}^{(i)}=0$ because recent studies of the lattice simulation [39] as well as chiral perturbation theory [40] imply negligible strange quark content. It has been pointed out [18] that the results of XENON100, CoGeNT and CRESST can be compatible, if the following relations are satisfied:

$$
\frac{f_{n}}{f_{p}} \simeq-0.7, \quad \sigma_{\mathrm{SI}}^{p} \simeq 2 \times 10^{-2} \mathrm{pb} .
$$

Note that $f_{n} \neq f_{p}$, and therefore the dark matter particle has isospin violating interactions with quarks.

\section{Model $S$}

For the model $\mathrm{S}$, there are two contributions to the effective coupling $\alpha_{q}$. One is from the exchange of the scalars $h_{1}$ and $h_{2}$, for which we find

$$
\alpha_{q}=-m_{q}\left(\frac{\lambda_{1} \sin \alpha}{m_{h_{1}}^{2}}-\frac{\lambda_{2} \cos \alpha}{m_{h_{2}}^{2}}\right),
$$


where we have assumed $m_{h_{1}}^{2} \ll m_{h_{2}}^{2}$, with $m_{h_{2}}$ being the SM(-like) Higgs boson mass. Note that $\alpha_{q} / m_{q}$ is independent of $q$, so that this contribution conserves the isospin. The other contribution is from the exchange of the Dirac fermions, $U$ and $D$ :

$$
\alpha_{q}=\frac{f_{U}^{2}}{2} \frac{m_{\phi}}{M_{U}^{2}-m_{\phi}^{2}} \delta_{q}^{u}+\frac{f_{D}^{2}}{2} \frac{m_{\phi}}{M_{D}^{2}-m_{\phi}^{2}} \delta_{q}^{d} \simeq \frac{f_{U}^{2}}{2} \frac{m_{\phi}}{M_{U}^{2}} \delta_{q}^{u}+\frac{f_{D}^{2}}{2} \frac{m_{\phi}}{M_{D}^{2}} \delta_{q}^{d},
$$

where we have assumed $m_{\phi}^{2} \ll M_{U, D}^{2}$. Clearly this contribution violates the isospin symmetry. For simplicity, let us assume $f_{D} \ll f_{U}$, and the total contribution is given by

$$
\begin{aligned}
\alpha_{q} & \simeq-m_{q} \frac{\lambda_{1} \sin \alpha}{m_{h_{1}}^{2}}+\frac{f_{U}^{2}}{2} \frac{m_{\phi}}{M_{U}^{2}} \delta_{q}^{u} \\
& =-m_{q} \frac{\lambda \cos \alpha \sin \alpha}{m_{h_{1}}^{2}}+\frac{f_{U}^{2}}{2} \frac{m_{\phi}}{M_{U}^{2}} \delta_{q}^{u} .
\end{aligned}
$$

Note that the existence of the two terms is crucial to realizing the opposite signs between $f_{p}$ and $f_{n}$, because the heavy quark $U$ (and also $D$ ) always positively contributes to $\alpha_{q}$.

Figure 1 shows the contours for various values of $\sigma_{\mathrm{SI}}^{p}$, along with the (red) straight line corresponding to the condition $f_{n} / f_{p}=-0.7$. The two conditions in Eq. (12) are satisfied for

$$
\frac{\sqrt{\lambda \cos \alpha \sin \alpha}}{m_{h_{1}}}=4.30 \times 10^{-2} \mathrm{GeV}^{-1}, \quad \frac{\left|f_{U}\right|}{M_{U}}=5.22 \times 10^{-3} \mathrm{GeV}^{-1} .
$$

Here we have fixed the dark matter mass as $m_{\phi}=8 \mathrm{GeV}$.

\section{Model F}

For the model $\mathrm{F}$, the effective coupling $\alpha_{q}$ is given by

$$
\begin{aligned}
\alpha_{q} & =-\frac{1}{2}\left[\sin 2 \theta_{u} f_{L} f_{R_{u}}\left(\frac{1}{M_{\tilde{U}_{1}}^{2}}-\frac{1}{M_{\tilde{U}_{2}}^{2}}\right) \delta_{q}^{u}+\sin 2 \theta_{d} f_{L} f_{R_{d}}\left(\frac{1}{M_{\tilde{D}_{1}}^{2}}-\frac{1}{M_{\tilde{D}_{2}}^{2}}\right) \delta_{q}^{d}\right] \\
& \simeq-\frac{1}{2}\left[\frac{\sin 2 \theta_{u} f_{L} f_{R_{u}}}{M_{\tilde{U}_{1}}^{2}} \delta_{q}^{u}+\frac{\sin 2 \theta_{d} f_{L} f_{R_{d}}}{M_{\tilde{D}_{1}}^{2}} \delta_{q}^{d}\right] .
\end{aligned}
$$

Here, for simplicity, we have taken a limit, $M_{\tilde{U}_{1}}^{2} \ll M_{\tilde{U}_{2}}^{2}$ and $M_{\tilde{D}_{1}}^{2} \ll M_{\tilde{D}_{2}}^{2}$. This effective coupling violates the isospin symmetry and $f_{n} / f_{p}<0$ can be realized when the relative signs between $\sin 2 \theta_{u} f_{R_{u}}$ and $\sin 2 \theta_{d} f_{R_{d}}$ are opposite. We further simplify the system by setting

$$
\begin{aligned}
& f_{L} \cos \theta_{u}=f_{R_{u}} \sin \theta_{u} \equiv f_{\tilde{U}}>0, \\
& f_{L} \cos \theta_{d}=-f_{R_{d}} \sin \theta_{d} \equiv f_{\tilde{D}}>0,
\end{aligned}
$$




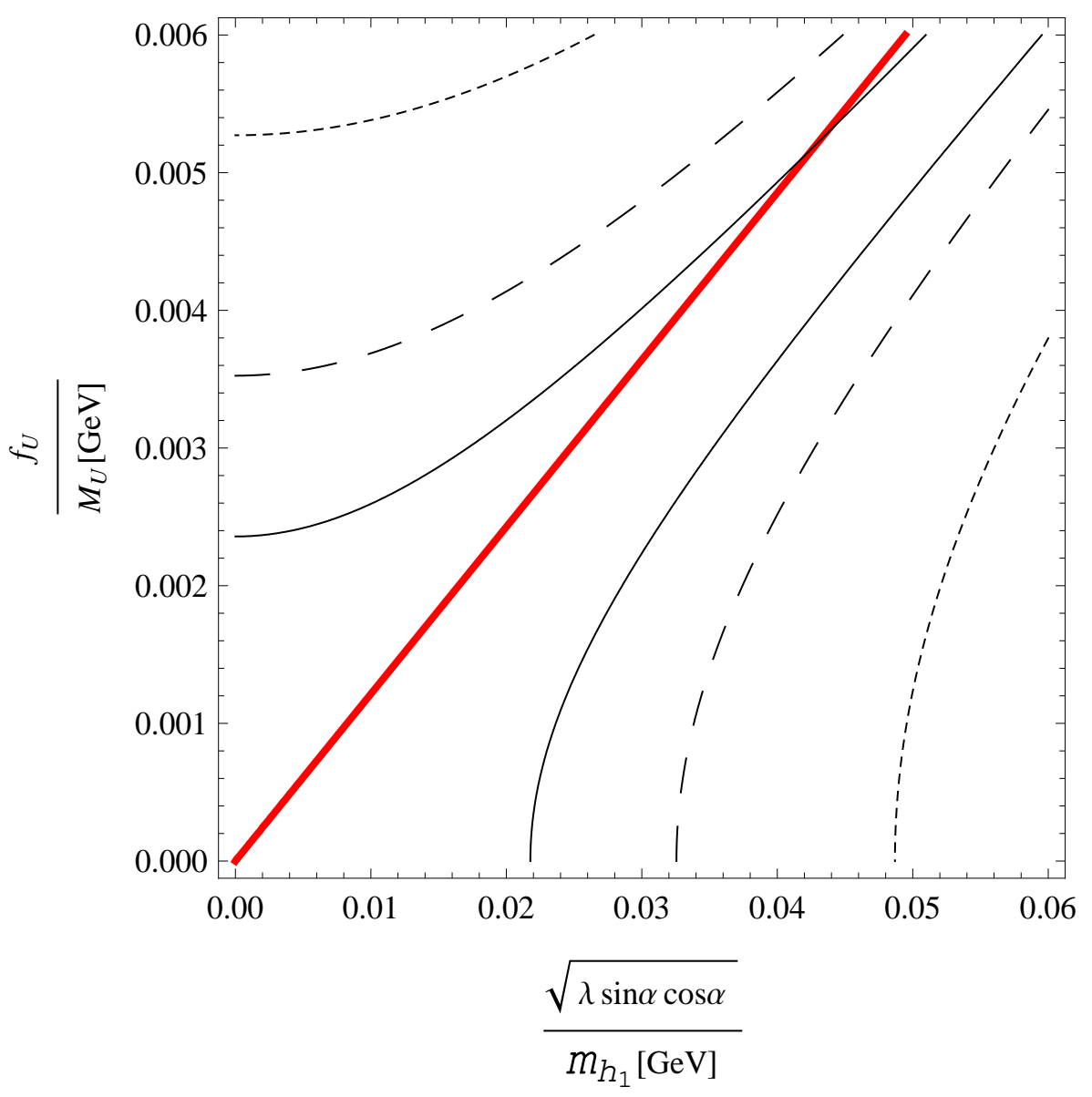

FIG. 1: The contours of scattering cross section with a proton for various values, $\sigma_{\mathrm{SI}}^{p}=0.02 \mathrm{pb}$ (solid), $0.1 \mathrm{pb}$ (dashed) and $0.5 \mathrm{pb}$ (dotted), together with the (red) straight line along which the condition $f_{n} / f_{p} \simeq-0.7$ is satisfied. Here we have fixed the dark matter mass as $m_{\phi}=8 \mathrm{GeV}$.

so that

$$
\alpha_{q} \simeq-\left(\frac{f_{\tilde{U}}}{M_{\tilde{U}_{1}}}\right)^{2} \delta_{q}^{u}+\left(\frac{f_{\tilde{D}}}{M_{\tilde{D}_{1}}}\right)^{2} \delta_{q}^{d}
$$

Figure 2 shows the contours for various values of $\sigma_{\mathrm{SI}}^{p}$, along with the (red) straight line corresponding to $f_{n} / f_{p}=-0.7$. The two conditions in Eq. (12) are satisfied for

$$
\frac{f_{\tilde{U}}}{M_{\tilde{U}_{1}}}=2.73 \times 10^{-3} \mathrm{GeV}^{-1}, \quad \frac{f_{\tilde{D}}}{M_{\tilde{D}_{1}}}=2.63 \times 10^{-3} \mathrm{GeV}^{-1}
$$




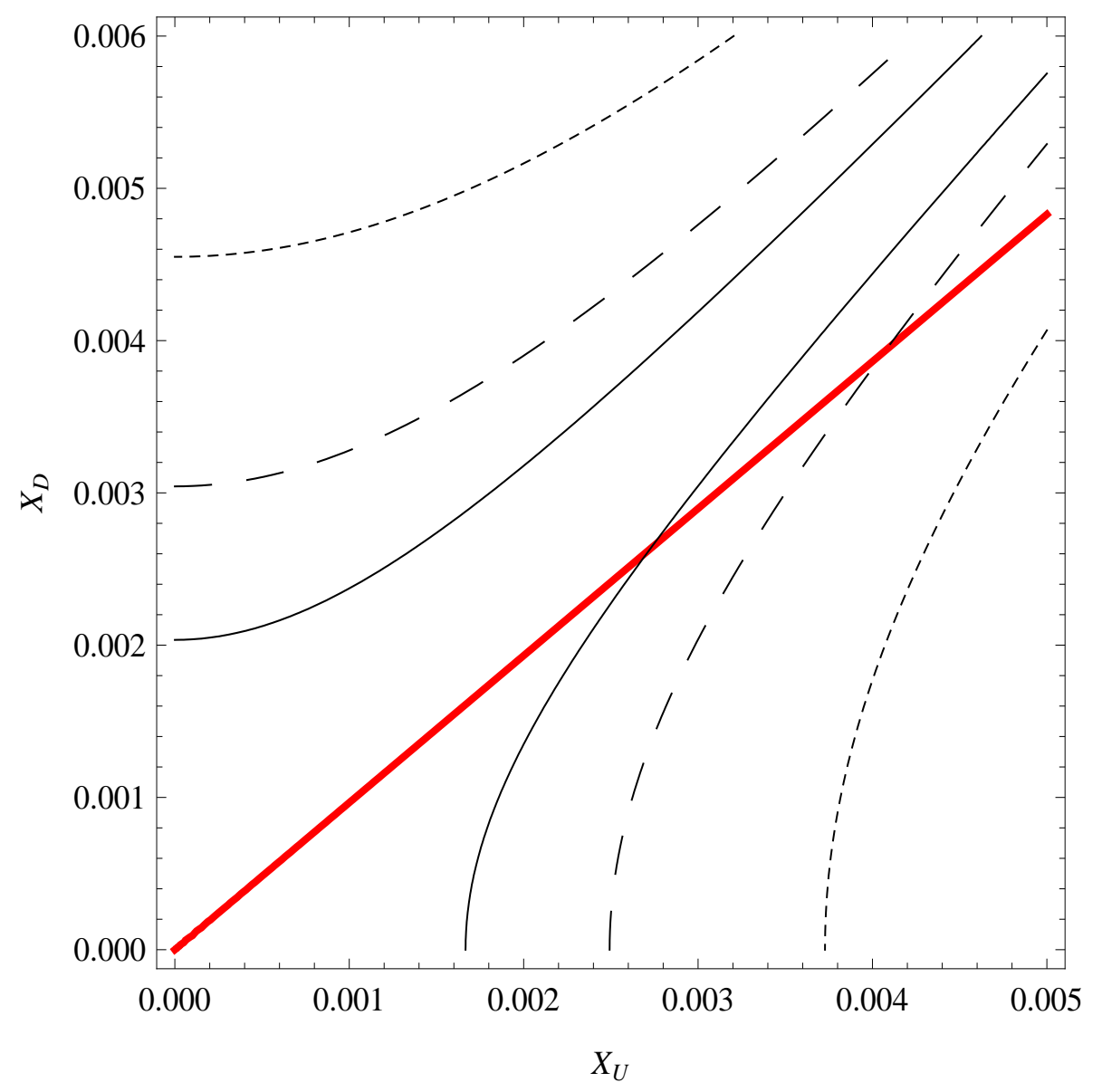

FIG. 2: The same as Fig. 1 but for the Dirac fermion dark matter. Here $X_{U} \equiv \frac{f_{\tilde{U}}}{M_{\tilde{U}_{1}}}$, and $X_{D} \equiv \frac{f_{\tilde{D}}}{M_{\tilde{D}_{1}}}$.

\section{DARK MATTER ANNIHILATION CROSS SECTION}

In this section, we estimate the annihilation cross section of the scalar/fermion dark matter particles for the parameters identified in the previous section to satisfy the conditions for the IVDM. We will see that the $s$-wave annihilation cross section of the dark matter is too large to reproduce the observed relic abundance. In order to achieve the correct relic abundance, one may consider a nonthermal dark matter scenario. However, this scenario cannot be viable, because the $s$-wave annihilation cross section already exceeds the upper bound obtained by the Fermi-LAT observations [17]. In the last part of this section, we will propose a simple scenario to realize the IVDM being consistent with the Fermi-LAT observations. 


\section{A. Model S}

The dominant dark matter annihilation process is found to be $\phi^{\dagger} \phi \rightarrow b \bar{b}$ mediated by the scalars, $h_{1}$ and $h_{2}$, in the $s$ channel. Assuming $m_{h_{1}}^{2} \lesssim m_{\phi}^{2} \ll m_{h_{2}}^{2}$, the $s$-wave annihilation cross section is evaluated as

$$
\langle\sigma v\rangle \simeq \frac{3}{16 \pi}\left(\frac{\lambda \sin 2 \alpha m_{b}}{4 m_{\phi}^{2}-m_{h_{1}}^{2}}\right)^{2},
$$

where $m_{b}=4.2 \mathrm{GeV}$ is the bottom quark mass. Using the values in Eq. (16), we find, for example, $\langle\sigma v\rangle \simeq 6.12 \mathrm{pb}$ for $m_{\phi}=8 \mathrm{GeV}$ and $m_{h_{1}}=2.9 \mathrm{GeV}$. This cross section is roughly one order of magnitude larger than the typical dark matter annihilation cross section $\langle\sigma v\rangle \simeq 1 \mathrm{pb}$ to achieve the observed relic density. Thus, in this case, the resultant dark matter abundance becomes too small. In order to realize the observed relic density, we may assume a nonthermal production of dark matter particles in the early Universe. However, this cannot be a phenomenologically viable scenario, because the dark matter annihilation cross section to the bottom quarks is constrained by the Fermi-LAT data as $\langle\sigma v\rangle \lesssim 0.5 \mathrm{pb}[17]$. In this case, the dark matter is overabundant and the relic density should be diluted by some mechanism in the history of the Universe. Since such a scenario is quite ambiguous, we do not consider it in this paper.

\section{B. Model F}

The $s$-wave annihilation modes are given by $t$-channel $\tilde{U} / \tilde{D}$ exchange with $u \bar{u} / d \bar{d}$ final states. In a limit $m_{\psi}^{2} \ll M_{\tilde{U}_{1,2}, \tilde{D}_{1,2}}^{2}$, the cross sections is found to be

$$
\langle\sigma v\rangle \simeq a_{u}+a_{d}
$$

with

$$
\begin{aligned}
a_{u}= & \frac{N_{c} m_{\psi}^{2}}{4 \pi}\left[f_{L}^{4}\left(\frac{\sin ^{2} \theta_{u}}{M_{\tilde{U}_{2}}^{2}}+\frac{\cos ^{2} \theta_{u}}{M_{\tilde{U}_{1}}^{2}}\right)^{2}+f_{R_{u}}^{4}\left(\frac{\cos ^{2} \theta_{u}}{M_{\tilde{U}_{2}}^{2}}+\frac{\sin ^{2} \theta_{u}}{M_{\tilde{U}_{1}}^{2}}\right)^{2}\right. \\
& \left.+\sin ^{2} 2 \theta_{u} f_{L}^{2} f_{R_{u}}^{2}\left(\frac{1}{M_{\tilde{U}_{1}}^{2}}-\frac{1}{M_{\tilde{U}_{2}}^{2}}\right)^{2}\right] \simeq \frac{3 N_{c} m_{\psi}^{2}}{2 \pi}\left(\frac{f_{\tilde{U}}}{M_{\tilde{U}_{1}}}\right)^{4} \\
a_{d}= & \frac{N_{c} m_{\psi}^{2}}{4 \pi}\left[f_{L}^{4}\left(\frac{\sin ^{2} \theta_{d}}{M_{\tilde{D}_{2}}^{2}}+\frac{\cos ^{2} \theta_{d}}{M_{\tilde{D}_{1}}^{2}}\right)^{2}+f_{R_{d}}^{4}\left(\frac{\cos ^{2} \theta_{d}}{M_{\tilde{D}_{2}}^{2}}+\frac{\sin ^{2} \theta_{d}}{M_{\tilde{D}_{1}}^{2}}\right)^{2}\right.
\end{aligned}
$$




$$
\left.+\sin ^{2} 2 \theta_{d} f_{L}^{2} f_{R_{d}}^{2}\left(\frac{1}{M_{\tilde{D}_{1}}^{2}}-\frac{1}{M_{\tilde{D}_{2}}^{2}}\right)^{2}\right] \simeq \frac{3 N_{c} m_{\psi}^{2}}{2 \pi}\left(\frac{f_{\tilde{D}}}{M_{\tilde{D}_{1}}}\right)^{4}
$$

where we have used Eq. (18) and the limit $M_{\tilde{U}_{1}}^{2} \ll M_{\tilde{U}_{2}}^{2}$ and $M_{\tilde{D}_{1}}^{2} \ll M_{\tilde{D}_{2}}^{2}$. Using the values in Eqs. (20), we find the annihilation cross section as

$$
\langle\sigma v\rangle \simeq 3.68 \mathrm{pb}
$$

which is too large to reproduce the correct thermal relic density of the dark matter particle in the present Universe. In order to make the relic abundance right, we may consider a nonthermal production of the dark matter particles in the early Universe. However, as in the model S, such a scenario is not viable by the Fermi-LAT observations [17]. The upper bound on the cosmic antiproton flux obtained by the Fermi-LAT observations is interpreted to a cross section upper bound of DM annihilations to up and down quarks as [29]

$$
\langle\sigma v\rangle \lesssim 0.2 \mathrm{pb}
$$

\section{Solution to too large annihilation cross section}

As we have seen, for a given parameter set to realize a large enough isospin violating scattering cross section with nuclei, the resultant annihilation cross section is too large to satisfy cosmological and astrophysical constraints. For relic density, one may assume a nonthermal dark matter production. However, as we have seen, such an idea cannot work because of the severe upper bound on the dark matter annihilation cross section from the Fermi-LAT observations. In order to avoid the Fermi-LAT constraints, we propose an extension of our model to the so-called "asymmetric dark matter" scenario [41 47]. This scenario is suitable to our model, because the global $U(1)_{G}$ symmetry introduced in our model leads to the conservation of the dark matter number. Once a suitable DM-antiDM asymmetry is created in the early Universe, the too large annihilation cross section nicely works to leave only the dark matter in the present Universe with the observed relic abundance. Since the relic abundance of antidark matter particles in the present Universe is much smaller than the dark matter one, a cosmic ray flux produced by DM and antiDM annihilations becomes much smaller and hence the constraint from the Fermi-LAT observations can be avoided. 
A relic density of the dark matter particles in the presence of dark matter asymmetry (chemical potential) has been analyzed in detail by solving the Boltzmann equations [48]. For example, with a suitable initial dark matter asymmetry, the observed relic abundance of the dark matter particle can be obtained by the $s$-wave annihilation cross section $\langle\sigma v\rangle=$ $\mathcal{O}(1) \mathrm{pb}$, while the relic abundance of antidark matter particle is found to be 2 orders of magnitude smaller than the dark matter one. As annihilation cross sections become larger, the relic abundance of anti-DM particle becomes exponentially smaller. This result is almost independent of WIMP dark matter mass. We apply the result to our scenario, so that the cosmic ray flux from DM-anti-DM pair annihilations is significantly suppressed and the constraint from the Fermi-LAT observations is avoided.

\section{CONSTRAINTS FROM COLLIDER EXPERIMENTS}

\section{A. Constraints on the mediator (s)quarks from LHC}

Our model includes heavy (s)quarks, which can be produced at the Large Hadron Collider (LHC) mainly through the gluon fusion process. The heavy (s)quarks, once produced, decay to the SM quarks and the dark matter particles, and this process is observed as the hadronic final states with transverse missing energy. Searches for such events have been performed at the LHC experiments, and the null result, so far, sets the lower bound on heavy (s)quark masses as $\gtrsim 800 \mathrm{GeV}$ [49]. This bound is obtained for the so-called simplified MSSM, where scalar quarks of the first two generations are produced at the LHC and decay to quarks and the lightest superpartner neutralino. Since we only introduced one generation of heavy (s)quarks, the mass bound on the mediator (s)quarks should be a little milder, but let us apply the bound for conservative discussion. From Eqs. (16) and (20), we can see that this mass bound is satisfied with the couplings being in a perturbative regime, $f_{U}^{2} /(4 \pi), f_{\tilde{U}, \tilde{D}}^{2} /(4 \pi) \ll 1$.

\section{B. Constraint from Higgs boson invisible decay}

In model $\mathrm{S}$, the scalar mass eigenstate $h_{2}$ is approximately identified as the SM Higgs boson. Through the mass mixing with the singlet scalar $s$, the SM Higgs boson decays to a 
pair of the dark matter particles. ${ }^{1}$ This decay width is given by

$$
\Gamma\left(h_{2} \rightarrow \phi \phi^{\dagger}\right)=\frac{\lambda^{2} \sin ^{2} \alpha v^{2}}{16 \pi m_{h_{2}}} \sqrt{1-\frac{4 m_{\phi}^{2}}{m_{h_{2}}^{2}}} .
$$

The current ATLAS [51] and CMS [52] data for the Higgs boson production and its various decay modes are mostly consistent with the SM expectations, and the branching ratio of an invisibly decaying Higgs boson is constrained (at $3 \sigma$ ) as [53]

$$
\mathrm{BR}\left(h_{2} \rightarrow \text { invisible }\right)=\frac{\Gamma\left(h_{2} \rightarrow \phi \phi^{\dagger}\right)}{\Gamma_{\mathrm{SM}}+\Gamma\left(h_{2} \rightarrow \phi \phi^{\dagger}\right)} \leq 0.35,
$$

where $\Gamma_{\mathrm{SM}}=4.07 \mathrm{MeV}$ [54] is the SM prediction of the total decay width of a Higgs boson with a $125 \mathrm{GeV}$ mass.

Using the result in Eq. (16), we can give the annihilation cross section of Eq. (21) and the Higgs invisible decay rate of Eq. (28) as a function of only $m_{h_{1}}$, with a fixed dark matter mass $m_{\phi}=8 \mathrm{GeV}$. The correlation between these two quantities is shown in Fig. 3 by varying $m_{h_{1}}$ in the range of $1 \mathrm{GeV} \leq m_{h_{1}} \leq 7.0 \mathrm{GeV}$. Here the vertical line denotes the upper bound, $\operatorname{BR}\left(h_{2} \rightarrow\right.$ invisible $)=0.35$ at $3 \sigma$ [53] while the horizontal line corresponds to a typical value $(\langle\sigma v\rangle=1 \mathrm{pb})$ of the WIMP dark matter annihilation cross section for reproducing the observed relic abundance. The upper bound $\operatorname{BR}\left(h_{2} \rightarrow\right.$ invisible $)=0.35$ is obtained by $m_{h_{1}} \simeq 2.9 \mathrm{GeV}$, for which we find the annihilation cross section $\langle\sigma v\rangle \simeq 6.1$ pb. Note that the asymmetric IVDM scenario we have proposed in the previous section can be consistent with the constraint on the Higgs invisible decay rate. In order for the asymmetric dark matter to be consistent with the observed relic abundance, we have a lower bound on the annihilation cross section as $\langle\sigma v\rangle \gtrsim 1 \mathrm{pb}[48]$. Applying this bound, we read $\operatorname{BR}\left(h_{2} \rightarrow\right.$ invisible $) \gtrsim 8 \%$ from Fig. 3. Precision measurements of Higgs decay width at future collider experiments such as the international linear collider, photon collider and muon collider can reveal the existence of the dark matter.

\section{CONCLUSIONS}

The IVDM scenario with destructive interference between the dark matter scatterings with a proton and a neutron offers an interesting possibility to reconcile conflicting results

1 This structure is the same as in the so-called Higgs portal dark matter scenario. See, for example, Ref. 50] for a detailed analysis and references therein. 


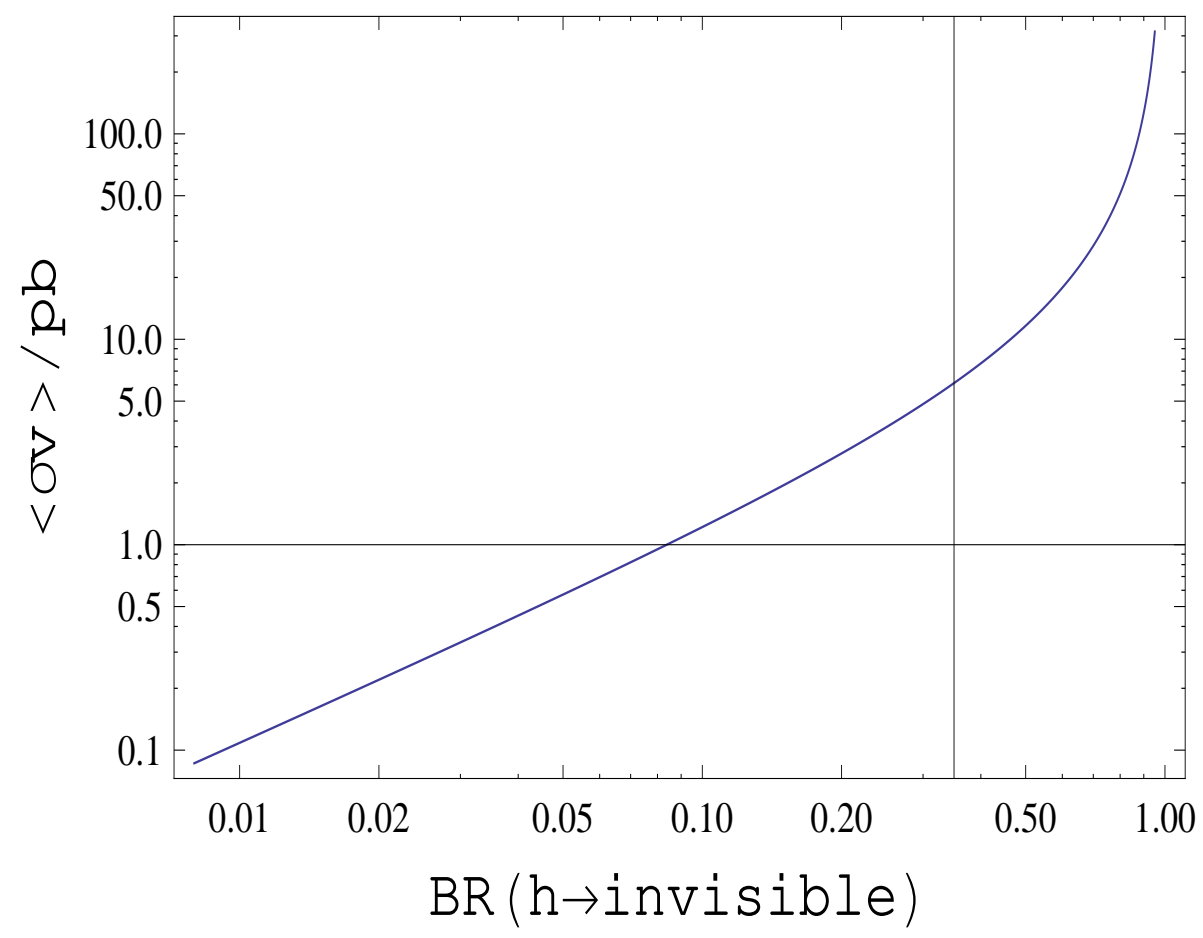

FIG. 3: The correlation between the Higgs invisible decay rate and the dark matter annihilation cross section through $m_{h_{1}}$. Here we have varied $m_{h_{1}}$ in the range of $1 \leq m_{h_{1}}(\mathrm{GeV}) \leq 7.0$.

among direct dark matter search experiments for a light WIMP with mass around $10 \mathrm{GeV}$. In this paper, we have considered two simple IVDM models and investigate various phenomenological aspects of the models, such as realization of the IVDM scenario, the constraints on dark matter annihilation cross sections from the dark matter relic abundance as well as an indirect search for dark matter, and collider experimental constraints on the extra particles introduced in our models.

One model introduces a complex scalar as a dark matter particle along with heavy extra quarks and a SM singlet real scalar, through which the dark matter particle couples with the SM up and down quarks. Isospin violating effective couplings are realized by the interference between processes mediated by the heavy quarks and the scalar. In the other model, we have introduced a Dirac fermion as a dark matter particle along with heavy colored scalars analogous to squarks in the MSSM, through which the dark matter particle couples with the SM quarks. The interference between two processes mediated by up-type squarks and down-type squarks realizes the isospin violating effective couplings. For both models, we 
have identified a parameter region suitable for the IVDM scenario. With the parameter regions, we have also calculated the relic abundance of the dark matter which is found to be too large to reproduce the observed relic abundance.

We have noticed that for both models, the calculated dark matter annihilation cross sections exceed the upper bound obtained by the Fermi-LAT observations too, and therefore the parameter regions for realizing the IVDM scenario are excluded. We have proposed a simple scenario to reconcile the IVDM scenario with the Fermi-LAT observations, namely, the IVDM being asymmetric. In our models, a global $U(1)_{\mathrm{G}}$ symmetry has been introduced whose conservation ensures the stability of a dark matter particle. At the same time, this global symmetry leads to the conservation of the dark matter number and this structure is suitable for the asymmetric dark matter scenario. As discussed above, we have found that the dark matter annihilation cross section is too large to satisfy cosmological and astrophysical constraints simultaneously. In fact, when a suitable asymmetry between dark matter-antidark matter is generated in the early Universe, the large cross section nicely works to leave only the dark matter in the present Universe. Thus, the relic abundance of the antidark matter particle is much less than the dark matter relic abundance; as a result, the flux of cosmic rays created by annihilations of the dark matter and antidark matter particles is dramatically suppressed and the constraint by the Fermi-LAT observations is avoided.

Since a variety of models to account for generating the dark matter asymmetry has been proposed (for an incomplete list, see e.g., [55 65]), we do not propose a specific model for it in this paper. However, we should note that some "dark matter number violating" operator, in other words the global $U(1)_{G}$ breaking terms, is necessary to generate the dark matter asymmetry in the Universe and such an operator might induce a dark matter number violating mass term at low energies, which must be sufficiently suppressed [66] not to spoil the asymmetric dark matter scenario. Concretely speaking, in model F, for instance, we may introduce the following scenario by means of a scalar condensate, which is analogous to the Affleck-Dine baryogenesis [67]. Although none of scalar fields carrying $U(1)_{G}$ charges develop vacuum expectation values at the present Universe, we may add the global $U(1)_{G}$ as well as the $C P$ violating potential, which is given as a function of the gauge invariant 
product, $\tilde{U}_{R} \tilde{D}_{R} \tilde{D}_{R}^{2}$, in the scalar potential. During the time that the Universe undergoes a false vacuum with nonvanishing expectation value ${ }^{3}$ of $\left\langle\tilde{U}_{R} \tilde{D}_{R} \tilde{D}_{R}\right\rangle$, dark matter asymmetry can be dynamically generated through the evolution of the coherent scalar in the similar way as the Affleck-Dine baryogenesis [67]. Note that although the global $U(1)_{G}$ symmetry is explicitly broken by terms with $\tilde{U}_{R} \tilde{D}_{R} \tilde{D}_{R}$, the model still possesses a residual $Z_{3}$ symmetry under which we may assign charges as $\tilde{Q}_{L}: \omega, \tilde{U}_{R}: \omega, \tilde{D}_{R}: \omega, \psi: \omega^{2}$, where $\omega=e^{i 2 \pi / 3}$. This $Z_{3}$ symmetry forbids a Majorana mass term for the dark matter. As above, in order not to induce the dark matter number violating mass term, the $U(1)_{G}$ breaking should arise via operators that respect a $Z_{N}$ subgroup of $U(1)_{G}$, with $N \geq 3$, independently of what mechanism actually generates the asymmetry. Then, this $Z_{N}$ symmetry forbids a dark matter number violating mass term for the dark matter.

We have also considered collider experimental constraints on our model. Colored fermions and scalars introduced in our models can be produced at the LHC and their decays to the SM quarks and dark matter particles yield the signal events with jets and missing transverse energy. We have confirmed that our IVDM scenario is realized consistently with the current LHC bound on the mass of the colored particles. In the model S, the SM Higgs boson invisibly decays to a pair of dark matter particles and the upper bound on the invisible decay rate is given by the LHC data. We have found a parameter region in which the IVDM scenario is consistent with the LHC bound on the Higgs boson invisible decay rate. Interestingly, our successful asymmetric IVDM scenario leads to a lower bound on the invisible decay rate about $8 \%$, so that precision measurements of the Higgs decay width at future collider experiments can test our scenario.

Observable effects of the asymmetric dark matter scenario in neutron stars have been investigated [68, 69]. Since the dark matter particles do not self-annihilate, once captured in neutron stars, dark matter particles are continuously accumulating and neutron stars eventually collapse into black holes. Observations of old neutron stars provide constraints on parameters of the asymmetric dark matter scenario. In particular, such constraints are

2 To be precise, this product should be like $\tilde{U}_{R} \tilde{D}_{R} \tilde{S}_{R}$, where an extra flavor of an additional down-type scalar quark $\left(\tilde{D}_{R}\right)$ has been introduced.

3 Here, we assume that our scalar potential has an appropriate form so that this direction is flat enough to develop an expectation value in the early Universe, while the existence of such a flat direction ( $D$-flat direction) is automatic for the original Affleck-Dine mechanism in the context of supersymmetric models. 
more severe for the case with a scalar dark matter because of the absence of Fermi degeneracy pressure. However, since the resultant constraints highly depend on the strength of dark matter self-interactions [69], we do not consider the constraints from the black formation in our scenario.

\section{Acknowledgments}

This work was supported in part by the DOE Grant No. DE-FG02-10ER41714 (N.O.), and by the scientific research grants from Hokkai-Gakuen (O.S). O.S would like to thank the Department of Physics and Astronomy at the University of Alabama for their warm hospitality where this work was initiated.

[1] R. Bernabei et al. [DAMA and LIBRA Collaborations], Eur. Phys. J. C 67, 39 (2010).

[2] C. E. Aalseth et al. [CoGeNT Collaboration], Phys. Rev. Lett. 106, 131301 (2011).

[3] C. E. Aalseth et al. [CoGeNT Collaboration], Phys. Rev. Lett. 107, 141301 (2011).

[4] G. Angloher et al., Eur. Phys. J. C 72, 1971 (2012).

[5] A. Brown, S. Henry, H. Kraus and C. McCabe, Phys. Rev. D 85, 021301 (2012).

[6] D. S. Akerib et al. [CDMS Collaboration], Phys. Rev. D 82, 122004 (2010);

Z. Ahmed et al. [CDMS Collaboration], Phys. Rev. Lett. 106, 131302 (2011).

[7] J. Angle et al. [XENON10 Collaboration], Phys. Rev. Lett. 107, 051301 (2011).

[8] E. Aprile et al. [XENON100 Collaboration], Phys. Rev. Lett. 107, 131302 (2011).

[9] E. Aprile et al. [XENON100 Collaboration], Phys. Rev. Lett. 109, 181301 (2012).

[10] M. Felizardo et al., Phys. Rev. Lett. 108, 201302 (2012).

[11] D. Hooper and T. Plehn, Phys. Lett. B 562, 18 (2003).

[12] A. Bottino, N. Fornengo and S. Scopel, Phys. Rev. D 67, 063519 (2003).

[13] D. G. Cerdeno, C. Hugonie, D. E. Lopez-Fogliani, C. Munoz and A. M. Teixeira, JHEP 0412, $048(2004)$.

[14] J. F. Gunion, D. Hooper and B. McElrath, Phys. Rev. D 73, 015011 (2006).

[15] D. G. Cerdeno, C. Munoz and O. Seto, Phys. Rev. D 79, 023510 (2009).

[16] D. G. Cerdeno, J. -H. Huh, M. Peiro and O. Seto, JCAP 1111, 027 (2011). 
[17] M. Ackermann et al. [Fermi-LAT Collaboration], Phys. Rev. Lett. 107, 241302 (2011).

[18] J. L. Feng, J. Kumar, D. Marfatia and D. Sanford, Phys. Lett. B 703, 124 (2011).

[19] R. Agnese et al. [CDMS Collaboration], arXiv:1304.4279 [hep-ex]].

[20] C. Kelso, D. Hooper and M. R. Buckley, Phys. Rev. D 85, 043515 (2012).

[21] M. T. Frandsen, F. Kahlhoefer, J. March-Russell, C. McCabe, M. McCullough and K. Schmidt-Hoberg, Phys. Rev. D 84, 041301 (2011).

[22] T. Schwetz and J. Zupan, JCAP 1108, 008 (2011).

[23] M. Farina, D. Pappadopulo, A. Strumia and T. Volansky, JCAP 1111, 010 (2011).

[24] C. McCabe, Phys. Rev. D 84, 043525 (2011).

[25] S. -L. Chen and Y. Zhang, Phys. Rev. D 84, 031301 (2011).

[26] Y. Gao, J. Kumar and D. Marfatia, Phys. Lett. B 704, 534 (2011).

[27] M. T. Frandsen, F. Kahlhoefer, C. McCabe, S. Sarkar and K. Schmidt-Hoberg, JCAP 1201, $024(2012)$.

[28] M. T. Frandsen, F. Kahlhoefer, C. McCabe, S. Sarkar and K. Schmidt-Hoberg, JCAP 1307, $023(2013)$.

[29] J. Kumar, D. Sanford and L. E. Strigari, Phys. Rev. D 85, 081301 (2012).

[30] H. -B. Jin, S. Miao and Y. -F. Zhou, Phys. Rev. D 87, 016012 (2013).

[31] J. M. Cline and A. R. Frey, Phys. Rev. D 84, 075003 (2011).

[32] X. Gao, Z. Kang and T. Li, JCAP 1301, 021 (2013).

[33] M. T. Frandsen, F. Kahlhoefer, S. Sarkar and K. Schmidt-Hoberg, JHEP 1109, 128 (2011).

[34] H. Kawase, JHEP 1112, 094 (2011).

[35] E. Del Nobile, C. Kouvaris, F. Sannino and J. Virkajarvi, Mod. Phys. Lett. A 27, 1250108 (2012).

[36] M. Acciarri et al. [L3 Collaboration], Phys. Lett. B 385, 454 (1996).

[37] R. Barate et al. [LEP Working Group for Higgs boson searches and ALEPH and DELPHI and L3 and OPAL Collaborations], Phys. Lett. B 565, 61 (2003).

[38] J. R. Ellis, K. A. Olive and C. Savage, Phys. Rev. D 77, 065026 (2008).

[39] K. Takeda et al. [JLQCD Collaboration], Phys. Rev. D 83, 114506 (2011).

[40] J. M. Alarcon, J. Martin Camalich and J. A. Oller, Phys. Rev. D 85, 051503 (2012).

[41] S. M. Barr, R. S. Chivukula and E. Farhi, Phys. Lett. B 241, 387 (1990).

[42] S. M. Barr, Phys. Rev. D 44, 3062 (1991). 
[43] D. B. Kaplan, Phys. Rev. Lett. 68, 741 (1992).

[44] S. D. Thomas, Phys. Lett. B 356, 256 (1995).

[45] D. Hooper, J. March-Russell and S. M. West, Phys. Lett. B 605, 228 (2005).

[46] R. Kitano and I. Low, Phys. Rev. D 71, 023510 (2005).

[47] D. E. Kaplan, M. A. Luty and K. M. Zurek, Phys. Rev. D 79, 115016 (2009).

[48] H. Iminniyaz, M. Drees and X. Chen, JCAP 1107, 003 (2011).

[49] S. Chatrchyan et al. [CMS Collaboration], arXiv:1301.2175 [hep-ex].

[50] S. Kanemura, S. Matsumoto, T. Nabeshima and N. Okada, Phys. Rev. D 82, 055026 (2010).

[51] G. Aad et al. [ATLAS Collaboration], Phys. Lett. B 716, 1 (2012).

[52] S. Chatrchyan et al. [CMS Collaboration], Phys. Lett. B 716, 30 (2012).

[53] G. Belanger, B. Dumont, U. Ellwanger, J. F. Gunion and S. Kraml, Phys. Lett. B 723, 340 (2013).

[54] A. Denner, S. Heinemeyer, I. Puljak, D. Rebuzzi and M. Spira, Eur. Phys. J. C 71, 1753 (2011).

[55] H. An, S. L. Chen, R. N. Mohapatra and Y. Zhang, JHEP 1003, 124 (2010).

[56] N. Haba and S. Matsumoto, Prog. Theor. Phys. 125, 1311 (2011).

[57] M. Blennow, B. Dasgupta, E. Fernandez-Martinez and N. Rius, JHEP 1103, 014 (2011).

[58] A. Falkowski, J. T. Ruderman and T. Volansky, JHEP 1105, 106 (2011).

[59] N. Okada and O. Seto, Phys. Rev. D 86, 063525 (2012).

[60] N. F. Bell, K. Petraki, I. M. Shoemaker and R. R. Volkas, Phys. Rev. D 84, 123505 (2011).

[61] C. Cheung and K. M. Zurek, Phys. Rev. D 84, 035007 (2011).

[62] B. von Harling, K. Petraki and R. R. Volkas, JCAP 1205, 021 (2012).

[63] J. March-Russell and M. McCullough, JCAP 1203, 019 (2012).

[64] K. Kamada and M. Yamaguchi, Phys. Rev. D 85, 103530 (2012).

[65] J. Unwin, JHEP 1306, 090 (2013).

[66] M. R. Buckley and S. Profumo, Phys. Rev. Lett. 108, 011301 (2012).

[67] I. Affleck and M. Dine, Nucl. Phys. B 249, 361 (1985).

[68] S. D. McDermott, H. -B. Yu and K. M. Zurek, Phys. Rev. D 85, 023519 (2012).

[69] J. Bramante, K. Fukushima and J. Kumar, Phys. Rev. D 87, 055012 (2013). 